\title{
Incidence of Tardive Dyskinesia with Risperidone or Olanzapine in the Elderly: Results from a 2-Year, Prospective Study in Antipsychotic-Naïve Patients
}

\author{
Margaret G Woerner', Christoph U Correll ${ }^{1,2}$, Jose Ma J Alvir ${ }^{3}$, Blaine Greenwald ${ }^{1,2}$, Howard Delman' \\ and John M Kane*,,
}

'The Zucker Hillside Hospital, North Shore — Long Island Jewish Health System, Glen Oaks, NY, USA; ${ }^{2}$ Department of Psychiatry, Albert Einstein College of Medicine, Bronx, NY, USA; ${ }^{3}$ Pfizer Pharmaceuticals, New York, NY, USA

\begin{abstract}
Tardive dyskinesia (TD) rates with second-generation antipsychotics (SGAs) are considered to be low relative to first-generation antipsychotics (FGAs), even in the particularly vulnerable elderly population. However, risk estimates are unavailable for patients naïve to FGAs. Therefore, we aimed to determine the TD incidence in particularly vulnerable, antipsychotic-naïve elderly patients treated with the SGA risperidone or olanzapine. The present work describes a prospective inception cohort study of antipsychotic-naïve elderly patients aged $\geqslant 55$ years identified at New York Metropolitan area in-patient and out-patient geriatric psychiatry facilities and nursing homes at the time of risperidone or olanzapine initiation. At baseline, 4 weeks, and at quarterly periods, patients underwent assessments of medical and medication history, abnormal involuntary movements, and extra-pyramidal signs. TD was classified using Schooler-Kane criteria. Included in the analyses were 207 subjects (age: 79.8 years, 70.0\% female, 86.5\% White), predominantly diagnosed with dementia (58.9\%) or a major mood disorder (30.9\%), although the principal treatment target was psychosis (78.7\%), with (59.4\%) or without (19.3\%) agitation. With risperidone $(n=159)$ the cumulative TD rate was 5.3\% (95\% confidence interval $(\mathrm{Cl}): 0.7,9.9 \%)$ after I year (mean dose: $1.0 \pm 0.76 \mathrm{mg} /$ day) and $7.2 \%(\mathrm{Cl}: 1.4,12.9 \%)$ after 2 years. With olanzapine $(n=48)$ the cumulative TD rate was $6.7 \%$ (Cl: 0 , 15.6\%) after I year (mean dose: $4.3 \pm 1.9 \mathrm{mg} /$ day) and $11.1 \%(\mathrm{Cl}: 0,23.1 \%)$ after 2 years. TD risk was higher in females, African Americans, and patients without past antidepressant treatment or with FGA co-treatment. The TD rates for geriatric patients treated with risperidone and olanzapine were comparable and substantially lower than previously reported for similar patients in direct observation studies using FGAs. This information is relevant for all patients receiving antipsychotics, not just the especially sensitive elderly.

Neuropsychopharmacology (20II) 36, 1738-1746; doi:I0.1038/npp.20 I I.55; published online 20 April 20II
\end{abstract}

Keywords: tardive dyskinesia; antipsychotics; olanzapine; risperidone; elderly; cohort study

\section{INTRODUCTION}

In the US, second-generation antipsychotic medications (SGAs) introduced after clozapine quickly became the treatment of choice for psychotic disorders, and have subsequently been used and approved for several nonpsychotic disorders as well. The widespread acceptance of SGAs, despite their increased cost, was based in part on their demonstrated reduced propensity to cause extrapyramidal side effects (EPS), such as parkinsonism and

*Correspondence: Dr JM Kane, Department of Psychiatry Research, The Zucker Hillside Hospital, 75-59 263rd Street, Glen Oaks, NY I 1004, USA, Tel: + I 718470 8|41, Fax: + I 7183437739 ,

E-mail: psychiatry@lij.edu or johnkaneny@hotmail.com

Received II November 20 10; revised 26 February 201 I; accepted 9 March 201। akathisia. Although there was less evidence for a decreased risk of SGAs for abnormal involuntary movements, particularly tardive dyskinesia (TD), a consensus quickly developed that this was also the case (Caroff et al, 2002; Casey, 1999; Jeste et al, 1999a, b; Marder et al, 2002; Kane et al, 1993; Dolder and Jeste, 2003).

However, the actual evidence for a low TD risk of SGAs is still limited, and, recently, advantages of SGAs vs firstgeneration antipsychotics (FGAs) regarding TD prevalence (de Leon, 2007) and incidence rates (Miller et al, 2008; Lee et al, 2005) have been questioned. Two recent systematic reviews pooled data on prevalence (Davidson et al, 2000) and incidence rates (Correll and Schenk, 2008; Correll et al, 2004) of TD or dyskinesia from studies using SGAs and FGAs that lasted at least 1 year. The review and comparison were restricted to studies conducted at the same time, that is, after introduction of non-clozapine SGAs, to reduce a 
potentially confounding cohort effect by studies conducted at different times, in different populations, and with different prevailing dosing and treatment strategies. In adults, both TD prevalence rates (13.1\% vs 32.4\%) (Correll and Schenk, 2008) and annualized incidence rates $(0.8 \%$ vs $5.4 \%$ (Correll et al, 2004) and 3.0\% vs 7.7\%) (Correll and Schenk, 2008) were significantly lower for SGAs than FGAs $(p<0.0001)$. The higher TD incidence rate for SGAs in the studies published since 2004 (Correll and Schenk, 2008) appeared to be attributable mainly to data from trials, where dyskinesia and not TD rates were reported. Restricting the analyses to studies reporting on TD cases yielded incidence rates of $1.2 \%$ (95\% confidence interval (CI): $0.9-1.5 \%)$ for SGAs and 3.9\% (95\% CI: $2.8-5.5 \%)$ for FGAs $(p<0.0001)$ (Correll and Schenk, 2008). Furthermore, low annualized TD incidence rates of only $0.42 \%$ using SGAs were also suggested by a review of 10 studies in youth $(n=783$, mean age: 9.7 years) treated mainly with risperidone (94\%) (Correll and Kane, 2007).

However, the validity of the data summarized above has also been challenged, raising concerns that only a minority of studies had been randomized, controlled trials directly comparing SGAs and FGAs; that FGA doses had been generally high; and that none of the studies had been designed specifically to assess incidence rates of TD (Correll et al, 2004). Moreover, results in favor of SGAs have not been uniform, with at least two adult studies failing to find an advantage for SGAs (de Leon, 2007; Miller et al, 2008).

Moreover, across the five naturalistic long-term studies in the most vulnerable elderly patient group, results have also been inconsistent. In the elderly, annualized TD incidence rates for SGAs ranged from 2.5\% (olanzapine (Kinon et al, 2005) and risperidone (Jeste et al, 2000)) and 2.7\% (quetiapine; Glazer et al, 1999) in patients with exclusive or predominant dementia, to $13.6 \%$ with risperidone (Davidson et al, 2000) in patients with mostly chronic schizophrenia, receiving high-dose risperidone, which exerted a dose effect on TD. In the 2004 review (Correll et al, 2004), no concurrent FGA TD rates in the elderly had been available. Therefore, TD rates of 5.3\% across the four available elderly studies $(n=479)$ were compared with historical TD rates using FGAs that were about 5-6 times higher (Jeste et al, 1995, 1999a, b; Saltz et al, 1991; Woerner et al, 1998). Since then, two additional studies reported on TD rates using SGAs and FGAs concurrently. Whereas in one study (mean age: 78 years) FGA-treated patients $(n=143 ; 5.5 \%)$ were more than twice as likely to develop TD than patients on olanzapine $(n=150 ; 2.5 \%)$ (Kinon et al, 2005), a large, pharmacoepidemiological study of elderly (mean age: 81 years) with predominant dementia reported almost identical incidence rates for SGAs $(n=9790 ; 3.5 \%=5.24 \%$ per 100 person-years $)$ and FGAs ( $n=12045 ; 3.0 \%=5.19 \%$ per 100 person-years) (Lee et al, 2005). However, these latter results are difficult to interpret, as patients were not assessed directly, TD caseness was based on chart diagnoses, and other, non-Parkinsonian, movement disorders were also included. Although chart diagnoses usually underestimate the true rates of side effects, this should have applied to both medication classes. An alternative explanation is that the background noise of non-antipsychotic-related movement disorders (related either to the underlying Alzheimer's disease or other illnesses) would have been considerably stronger, thereby diluting potential differences in the low-specificity, chart diagnosis-based rates of TD.

Similarly, a recent naturalistic 4-year cohort study of 352 adult and elderly patients (age range: $18-78$ years, mean: 42 years) with schizophrenia, schizoaffective disorder, and affective disorders suggested that there is no significant difference between the annualized TD incidence rates in patients receiving SGAs (5.9\%) or FGAs (5.6\%) using the Glazer-Morgenstern criteria (Woods et al, 2010). However, this study classified FGA or SGA users only based on the last 6 months of study participation. This methodology makes results vulnerable to carry over effects or treatment selection effects, in that patients with emergent TD on FGAs were possibly switched preferably to SGAs.

In summary, the recent TD incidence data for SGAs are restricted predominantly to studies that were not designed to focus explicitly on TD. In addition, the quality of assessment methods, frequency, rater training and reliability, and the criteria for designating a case differed widely. Most importantly, subjects had varying lengths of treatment, including FGA treatment prior to baseline. Moreover, the extent to which there was a history of dyskinesia, or in some studies, abnormal movements at baseline, is also unclear. To date, there are no long-term, prospective studies that have directly assessed TD rates using SGAs exclusively in antipsychotic-naïve subjects.

Therefore, the current study was specifically designed to provide an estimate of TD risk associated with two of the most commonly used SGAs, risperidone and olanzapine. In addition, our previous work (Woerner et al, 1998) had shown that the risk of TD with FGAs is 3-5 times higher among patients aged $\geqslant 55$ years than in younger patients, and we wanted to assess the TD risk of two commonly used SGAs in a similar population and design. Thus, we again studied older individuals to take advantage of this 'enriched' sample, in which the impact of drugs is magnified, enabling us to answer the question in a relatively short time frame and with a smaller sample size than might otherwise be required. Furthermore, we focused on antipsychotic-naïve subjects so that the TD risk could be attributed to the medications the patients received in a prospective, naturalistic setting.

Based on the pharmacology of risperidone and olanzapine, and the limited data available at the time, we hypothesized that the TD rates for olanzapine and risperidone would be significantly lower than the rates found for FGAs in our previous study of antipsychoticnaïve elderly patients, (Saltz et al, 1991), and that, based on lower EPS liability, the TD rate for olanzapine would be lower than for risperidone.

\section{MATERIALS AND METHODS}

\section{Subjects}

Patients 55 years or older who were just beginning their first antipsychotic treatment with either risperidone or olanzapine were recruited from the geriatric services of The Zucker Hillside Hospital and Winthrop University Hospital, the Geriatric Medical Group of North Shore University Hospital, The Hebrew Home for the Aged, The Gurwin 
Jewish Geriatric Center, and St John's Episcopal Hospital and Nursing Home. All facilities are located in New York City or Nassau County, New York. The study was approved and monitored by the Institutional Review Board (IRB) of the North Shore Long Island Jewish Health System and also by the IRBs of participating facilities not affiliated with the Health System. When the study began, only two participating sites required written informed consent (The Hebrew Home for the Aged and St John's Episcopal Hospital and Nursing home). For the other sites, the treating physician's permission was obtained prior to explaining the study to patients and family members; verbal agreement to participate was obtained and documented in the research chart. During the final year of the study, written informed consent was obtained for all new subjects as well as those continuing in follow-up, as a result of changes in IRB directives. Consent was obtained from patients who were considered competent by their physicians, but family members were informed as well. A family member gave formal consent for patients not clearly regarded as competent. Separate written informed consent was obtained for videotaping, and written releases were obtained for records of prior treatment. Initial assessments were performed at the site of recruitment; follow-up assessments took place wherever patients were residing (home visits and visits to nursing home placements were the rule).

Patients with abnormal involuntary movements at their initial examination were excluded from the analyses, as were those with a previous history of antipsychotic treatment or with neurological disorders (Huntington's or Parkinson's disease), which may have produced abnormal movements, and those who did not have at least one followup examination.

\section{Assessments}

The following were completed at study entry: medical record reviews and interviews with patients, relatives, and treatment staff to obtain demographic, medical, and psychiatric histories, and a standardized examination for abnormal involuntary movements and EPS, with ratings on the modified Simpson Dyskinesia Scale (SDS) (Simpson et al, 1979) and the Simpson-Angus Extrapyramidal Side Effect Scale (SAEPS) (Simpson and Angus, 1970). The SDS and SAEPS ratings and treatment reviews were repeated at 4 weeks and then quarterly. Psychiatric and medical diagnoses were obtained from clinical records; the criteria of the Diagnostic and Statistical Manual of Mental Disorders, Fourth Edition (DSM-IV) (American Psychiatric Association, 1987) were used at each study site. For cases where more than one diagnosis was listed, the records were reviewed and a judgment was made to designate a primary diagnosis.

The SDS includes 28 items and a six-point global score. In addition, the seven body-area items of the Abnormal Involuntary Movements Scale (AIMS) (Guy, 1976) were rated. Refresher training for raters was conducted every 3 months to minimize rater drift. Inter-rater reliability was tested annually; the intra-class correlation coefficient was 0.92 on the SDS global rating and 0.92 for the sum of seven AIMS ratings for the team of four trained raters at the last testing. Raters were 'blind' to patients' medications; they were not involved in treatment decisions and rated patients without checking treatment status or medical records prior to the TD assessments.

Patients with a global score of 1 ('questionable') or greater were examined by a second rater and, in some cases, a third rater to confirm the severity level. A global rating of at least 2 ('mild'), by two independent examiners, was required to designate a 'case' of TD. For all of the TD cases, severity criteria according to the Research Diagnosis of Tardive Dyskinesia (Schooler and Kane, 1982) were also fulfilled; these require AIMS ratings of 'mild' movements in two or more body areas, or 'moderate' or greater in one. Whenever possible, patients with presumptive TD were videotaped. We did not participate in treatment decisions, but informed patients and treatment staff of our findings.

\section{Statistical Methods}

We estimated the cumulative rates of TD using life table analysis, and used Cox proportional hazards regression to examine the effects of single and multiple potential risk factors. The time to TD development was calculated starting from the day of the first antipsychotic dose, whether or not the patient had been examined on that date.

We used survival analysis because of the different lengths of follow-up for the patients. The majority of patients did not develop abnormal movements during follow-up, leading to right-censored data, where the time to event is unknown (having not yet occurred) but is known to be at least as long as the follow-up period. Survival analysis adjusts for rightcensored data. The LIFETEST procedure was used for life table analysis (SAS Institute, 1989). Cox proportional hazards regression models were run using the PHREG procedure of SAS (SAS Institute, 1994). Categorical variables (eg, medication, race) were treated as strata in survival analyses using the LIFETEST procedure. Continuous variables (eg, age) were assessed using Cox regression models.

Patients who received more than two continuous weeks of conventional antipsychotic drugs during the study were considered censored observations as of the beginning of the 2 -week period. Some patients had repeated assessments long after they stopped taking antipsychotic medication. As antipsychotic drugs may, paradoxically, have a masking effect on dyskinesia, a 100-day drug-free window was allowed where patients were considered still at risk for developing TD. If patients who stopped and remained off antipsychotic drugs did not develop TD within 100 days, their data were right-censored at the end of the 100 days. Thus, any dyskinesias diagnosed more than 100 days after discontinuation of antipsychotic medication would not contribute to the $\mathrm{TD}$ rate.

Three sets of survival analysis were performed: 1 . Analyses including all 207 subjects (combining those who started on olanzapine and risperidone); 2 . Separate analyses for the 159 subjects who started on risperidone and the 48 who started on olanzapine (regardless of later switches to a different second-generation drug); and 3. Analyses using 'pure' groups consisting of individuals who began treatment with olanzapine and risperidone; for these two analyses, observations were censored either upon start of a different medication or 100 days after ending the original drug. This 
provides cumulative TD rates based on treatment with only one single antipsychotic.

\section{RESULTS}

\section{Sample}

Baseline examination was conducted on 404 patients. For analyses of TD rates and risk factors, we included patients who were treated with risperidone or olanzapine for $\geqslant 7$ days and were followed up for $\geqslant 4$ weeks. One hundred ninety-seven patients failed these criteria: 46 never started antipsychotics or had $<1$ week of exposure; nine died; 52 refused follow-up; 53 were excluded because information indicating substantial prior antipsychotic treatment was later obtained; 18 moved out of the area, or could not be contacted; and eight could not be examined for medical and other reasons.

In addition, 11 patients had abnormal movements at baseline and were excluded from the analyses; five of them were found to have had prior antipsychotic treatment. A total of 131 patients were lost either before completing 2 years of follow-up or before the end of the study: 43 patients died; 49 refused further ratings; 15 became too incapacitated to continue; and 24 moved or were withdrawn for other reasons.

\section{Subject Characteristics}

Patients were on average 79.8 years old; $70.0 \%$ were female and $86.5 \%$ were White (Table 1). The most frequent diagnoses were dementia (58.9\%) or a major mood disorder $(30.9 \%)$. The most frequent indication for antipsychotic treatment was psychosis $(n=163(78.7 \%))$, with $(n=123$ $(59.4 \%))$ or without $(n=40(19.3 \%))$ agitation. Thirty-seven patients $(18 \%)$ had agitation without psychosis and seven (3\%) were not described as either psychotic or agitated at baseline. Patient characteristics were largely similar across the two groups. Exceptions included that, compared with risperidone-treated patients, olanzapine-treated patients were significantly more likely to be male, younger, and more likely to be treated for psychosis ( $90 \%$ vs $75 \%$, $\left.\chi^{2}=3.58, \mathrm{df}=1, p=0.044\right)$.

There were no significant differences between the groups in terms of medical histories, which included the following conditions: cardiovascular disorder, 141 (68\%); endocrine disorder, 74 (36\%); musculoskeletal and connective tissue disease, 54 (26\%); nervous system disorder, 48 (23\%); gastrointestinal disorders, 41 (20\%); genitourinary disorder, 28 (14\%); cancer, 22 (11\%); and respiratory disease, 22 (11\%). Ninety-six percent of the patients had at least one medical condition and $75 \%$ had at least two conditions.

\section{Antipsychotic Treatment during the Study}

Perhaps because it was approved for marketing earlier, and became the primary drug of choice at our study sites, more subjects started treatment with risperidone $(n=159)$ than olanzapine $(n=48)$. Fifty-seven $(27.5 \%)$ of the 207 subjects (one quarter of the risperidone and one-third of the olanzapine subjects) were switched to a different antipsychotic for at least 2 weeks during the follow-up period. Most of the switches $(45 / 57,79 \%)$ were to a different SGA, the remainder to haloperidol $(n=7)$ or another conventional drug $(n=5)$. The risperidone and olanzapine groups did not differ in terms of the proportion of subjects who had some exposure ( $<2$ weeks) to a conventional agent: $22 / 159$ $(23 \%)$ of the risperidone subjects and $6 / 48(21 \%)$ of the olanzapine subjects. Although treatment tended to be intermittent and/or short term, 199 (96\%) of the patients were treated continuously for the first month of treatment. Of the 126 patients followed for 1 year, 62 (49\%) were treated for 49 weeks or more during the year, and $10(8 \%)$ for 2 months or less.

Medication dosage was very low for most patients. The mean (SD) daily dose for the first 2 weeks of treatment was $0.84(0.48) \mathrm{mg}$ for the 159 patients on risperidone and 4.80 (2.2) $\mathrm{mg}$ for the 48 patients on olanzapine. The mean (SD) daily dose was $1.04(0.76) \mathrm{mg}$ at 1 year for the 53 patients on risperidone and 4.26 (1.93) for the 17 patients on olanzapine at 1 year. Thirty patients were treated with anticholinergic medication, most for short periods and in low doses, without differences between the risperidone and olanzapine groups.

\section{Dyskinesia Cases Identified During Follow-up}

Thirteen patients developed TD during follow-up. Of them, four developed TD after more than 2 weeks of FGA treatment and were considered as censored observations in our analyses, leaving nine cases occurring during the above-defined 'period at risk', and included in our analyses. All nine cases were rated as 'mild' at the time of diagnosis: severity of TD was never rated more than 'mild' for seven (78\%); two cases (22\%) were rated moderate in severity at some point during their follow-up; and no cases were rated more than moderate at any time. Orofacial movements predominated and were always present whenever any movements were observed; five patients $(56 \%)$ had only orofacial movements throughout. The movements persisted for at least 3 months in five (56\%) of the cases and for at least 6 months in two (22\%) cases. There were three cases of transient dyskinesia (movements remitted within 3 months). Persistence was indeterminate for one case that was not followed for 3 months.

\section{TD Rate}

Table 2 illustrates the cumulative proportion of cases occurring and of the original sample remaining at risk for TD over time for all 207 subjects. Table 2 presents the rates for the separate analyses. No new cases were observed after 79 weeks in the risperidone group or after 68 weeks in the olanzapine group. The TD rates for the two SGAs did not differ significantly in any of the analyses. Controlling for age and presence of psychosis, and limiting the analyses to women did not affect the non-significant comparison between risperidone and olanzapine.

\section{Risk Factors for TD Development}

Small number of cases makes analysis of risk factors problematic, especially for multivariate analyses. Peduzzi et al (1996) have recommended at least 10 events per 
Table I Subject Characteristics at Study Entry

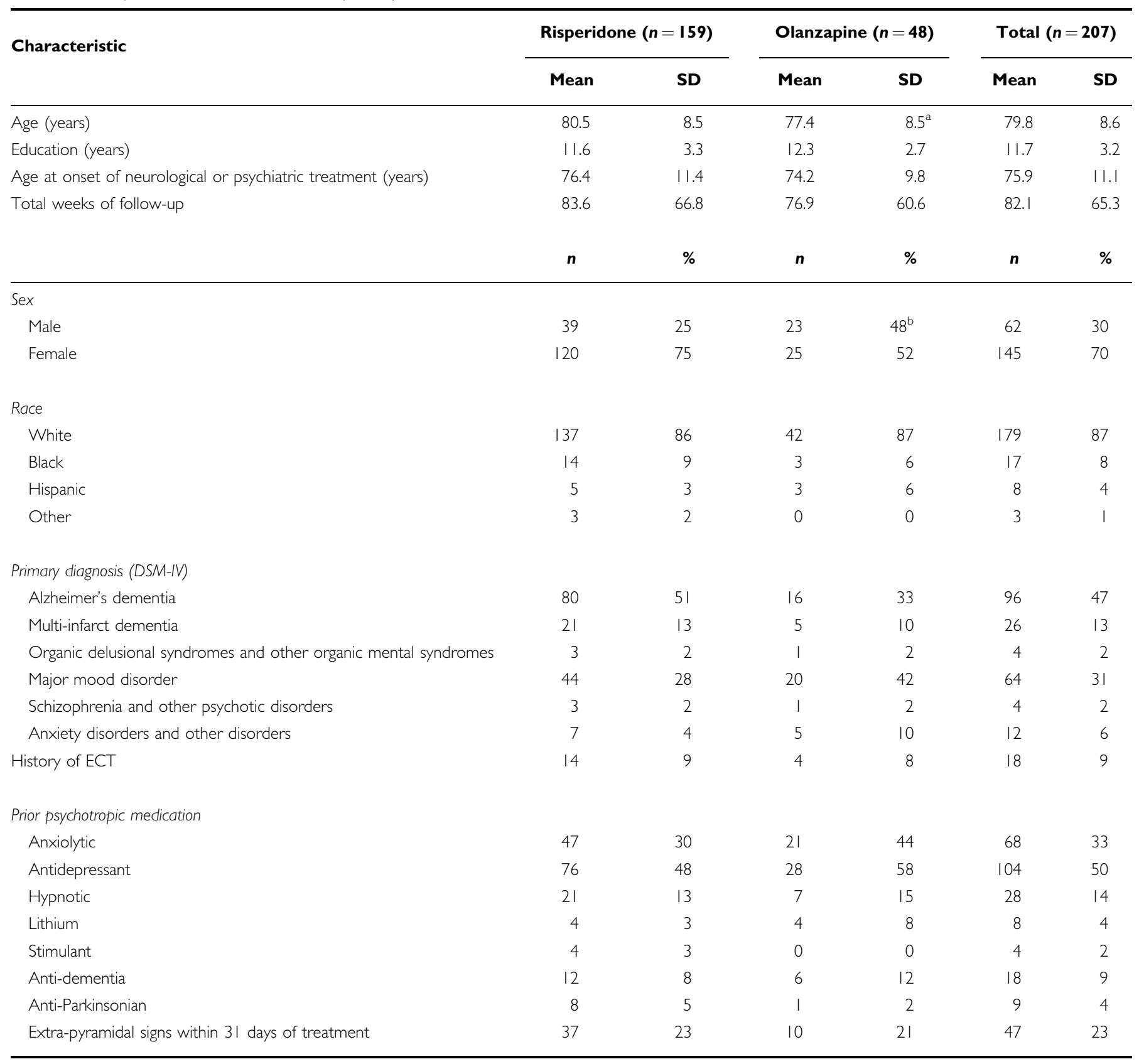

${ }^{a} t=2.23, d f=205, p=0.027$ (risperidone vs olanzapine subjects).

${ }^{b}$ Fisher's exact test, two-sided probability $=0.044$ (risperidone vs olanzapine subjects).

Cumulative Rate of TD for Elderly Subjects Treated with SGA Drugs.

covariate in logistic regression problems. However, because of the potential clinical importance of identifying risk factors, we examined a number of patient and treatment characteristics as potential predictors of TD development, and found higher rates of TD among women, African Americans, and patients who had no history of antidepressant medication. All of the nine TD cases that were included in the analyses occurred among women, resulting in cumulative incidence rates (and 95\% CIs) of $7.5 \%$ (2.1, $13.0)$ at 42 weeks and $10.8 \%(3.9,17.7)$ at 79 weeks for women $\left(\log \operatorname{rank}(\mathrm{LR}) \chi^{2},(\mathrm{df}=1)=3.12, p=0.08\right)$.
African Americans had higher rates compared with other racial groups. Although there were only 17 study subjects who were of African American descent, four of the nine cases occurred among African Americans, resulting in cumulative incidence rates (and 95\% CIs) of $31.3 \%$ (1.3, $61.2)$ and $45.0 \%(11.0,79.0)$ at 42 and 79 weeks of follow-up, respectively, compared with the maximum rate of $4.6 \%$ $(0.5,8.7)$ in other racial groups after 68 weeks of follow-up $\left(\mathrm{LR} \chi^{2},(\mathrm{df}=1)=16.7, p<0.0001\right)$.

Patients with a history of treatment with antidepressant drugs were less likely to develop TD. There was an almost 
Table 2 Cumulative TD Rates

\begin{tabular}{|c|c|c|c|c|c|}
\hline \multirow{2}{*}{ Group } & \multirow[b]{2}{*}{$n$} & \multicolumn{2}{|c|}{ I year } & \multicolumn{2}{|c|}{2 year } \\
\hline & & Rate (\%) & $95 \% \mathrm{Cl}$ & Rate (\%) & $95 \% \mathrm{Cl}$ \\
\hline Total & 207 & 5.7 & $1.5,10.0$ & 8.2 & $2.9,13.4$ \\
\hline All risperidone & 159 & 5.3 & $0.7,9.9$ & 7.2 & $1.4,12.9$ \\
\hline All olanzapine & 48 & 6.7 & $0.0,15.6$ & 11.1 & $0.0,23$ \\
\hline 'Pure' risperidone & 159 & 4.7 & $0.0,9.5$ & 7.1 & $0.6,13.7$ \\
\hline 'Pure' olanzapine & 48 & 4.0 & $0.0,11.8$ & 10.4 & $0.0,25$ \\
\hline
\end{tabular}

equal number of patients with $(n=104)$ and without $(n=103)$ history of antidepressant treatment, but eight of the nine cases occurred among the group without such history. The only TD case in the group with antidepressant treatment history occurred at 39 weeks, resulting in a cumulative rate of $1.8 \%(0.0,5.2)$. The cumulative rates in the group without antidepressant treatment history were $9.8 \%(2.2,17.4)$ at 42 weeks and $14.5 \%(4.9,24.0)$ at 79 weeks $\left(\operatorname{LR} \chi^{2},(\mathrm{df}=1)=5.97, p=0.02\right)$.

Although dose of antipsychotic medication during the study period was not significantly related to the development of TD, patients who were ever treated with an FGA were significantly more likely to develop TD. Note that patients' data were censored at the point where conventional treatment for more than 2 weeks began; thus this finding represents the effect of FGA treatment for a total of 1-13 days. This treatment usually occurred at the beginning of the inpatient stay and was prescribed PRN for agitation. Only 25 patients had some FGA exposure prior to their last observation in the analyses. However, these 25 patients included four of the nine cases of TD that occurred (LR $\chi^{2}$, $(\mathrm{df}=1)=6.79, p<0.01)$.

\section{DISCUSSION}

Our findings in the first prospectively followed, antipsychotic-naïve sample confirm the early consensus that SGAs are associated with a relatively low risk of TD and that this risk is considerably lower than that observed with FGAs in studies where patients were assessed directly. Our data are limited to olanzapine and risperidone, but there appears to be little reason to expect major differences for the newer SGAs as we did not find different TD incidence rates for risperidone and olanzapine, which differ in their potential to cause acute EPS. Our previous study findings in 261 antipsychotic-naïve geriatric patients treated with FGAs and followed with the same methodology indicated cumulative TD rates of $20 \%$ (95\% CI: $14-26 \%$ ) after 1 year, $30 \%(95 \%$ CI: $22-38 \%$ ) after 2 years, and 42\% (95\% CI: 32-53\%) after 3 years of follow-up (Woerner et al, 1998). Importantly, the TD incidence rate differences found in this study with risperidone and olanzapine are lower not only numerically but also in a very clinically meaningful way, translating into numbers-needed-to-treat of 7 to avoid one additional case of TD at the end of 1 year $(20-5.7 \%=14.3 \% ; 1 / 0.143=6.99)$ and of 5 at the end of year- $2 \quad(30-8.2 \%=21.8 \%$; $1 / 0.218=4.59)$. Moreover, the current study results indicate a maximum rate of $8.2 \%(95 \% \mathrm{CI}=2.9-13.4 \%)$ at 79 weeks of follow-up, with no additional cases occurring after that. This indicates that elderly may be at highest risk for development of TD in the first 18-24 months. It is also noteworthy that TD cases were observed after as little as 2 weeks of FGA treatment in the earlier study, whereas no cases were observed prior to 12 weeks of SGA treatment in the current study. This is relevant because of the not infrequent short-term use of FGAs for agitation or delirium. More cases occurring with FGAs were rated as 'moderate' or higher in severity, and more were persistent for 3 or 6 months. While we do not have a concurrent comparison of FGAs vs SGAs, the design and methodology for the two studies were identical, the raters for both studies were required to demonstrate reliability with the same expertrated training videotapes, and the sample characteristics for the two studies are very similar, allowing us to be relatively confident in the reliability of the differences between the rates. Furthermore, the chlorpromazine equivalent doses in the two studies were also very similar. The FGA dose in the previous study was $80 \pm 156 \mathrm{mg} /$ day, being $\leqslant 50$-mg chlorpromazine equivalents for $51 \%$ of patients at 1 year (which is equivalent to $\leqslant 1 \mathrm{mg}$ haloperidol) (Woerner et al, 1998). Likewise, the mean risperidone dose and corresponding chlorpromazine equivalents (Woods, 2003) were $1.04 \pm$ $0.76 \mathrm{mg}$ and $52.0 \pm 38.6 \mathrm{mg} /$ day, respectively, and the olanzapine dose and corresponding chlorpromazine equivalents were $4.26 \pm 1.93 \mathrm{mg}$ and $85.2 \pm 38.6 \mathrm{mg} /$ day, respectively.

The results of lower risk for SGAs compared with FGAs in older populations are consistent with two other shorter term trials in mixed adult and elderly populations. In one 9-month, open-label study of 61 adults aged $>45$ years (mean age $=66$ years) with schizophrenia, mood disorders, dementia, and organic mental disorders, cumulative TD incidence rates were significantly lower with risperidone (median dose $=1.0 \mathrm{mg} /$ day) compared with that in haloperidol-treated comparison subjects (median dose $=1.0$ mg/day), matched for age, sex, and duration of FGA treatment (5vs 32\%, $p<0.05$ ). In addition, results are also consistent with an important and unique naturalistic study that included a high-risk population of middle-aged (aged $\geqslant 45$ years) and elderly patients with predominantly psychotic disorders $(n=240)$ who had 'borderline TD', defined by presence of a mild severity rating on one AIMS item. Patients treated with FGAs ( $n=130$, mean age: 60 years) were approximately twice as likely to develop definitive TD during the 6-month study period compared with those treated with SGAs ( $n=110$, mean age: 65 years) $(p<0.001)$. This difference was found, although patients on SGAs were significantly older and had more severe EPS at baseline.

In contrast to our expectations, TD rates were very similar for risperidone and olanzapine, even when we looked at the 'pure' analyses that included patients only for the time they were treated with their original antipsychotic. Our expectation that higher rates would be found for subjects treated with risperidone was based on our own and others' case reports, as well as the evidence that risperidone produces EPS in a dose-related manner, and based on the similarity of olanzapine's receptor profile to that of clozapine. Although we found no evidence for an effect of dose on TD risk in this study where doses were very low, resulting in comparable EPS rates for these two SGAs, it is possible that differences between the drugs might emerge at 
higher dose levels when one would also expect to see a greater differentiation in EPS liability. This is suggested by results from one study in the elderly where there was a dose-dependent TD risk (Jeste et al, 2000). It must be emphasized that interpretation of the comparisons between the TD rates for the two antipsychotics is constrained by the sample size and the fact that the drugs were not randomly assigned. It is possible that subjects considered to be at risk for motor side effects were more likely to be prescribed olanzapine. This might have inflated the rate of TD in the olanzapine group, relative to the risperidone group. On the other hand, we found that subjects treated with olanzapine were younger, and included a higher proportion of males as well as patients with psychosis. Thus, the age and sex differences might have led to an underestimation of the relative rate for the olanzapine subjects. Moreover, our results are consistent with a prior review of 1-year TD rates in non-elderly adults where the TD risk for risperidone and olanzapine was also found to be comparable, ranging from 0 to $0.7 \%$ per year with risperidone and 0 to $0.5 \%$ per year for olanzapine (Correll et al, 2004).

Although we had limited power to investigate risk factors for TD development, higher rates for African Americans and women were salient in our study. Although we found no evidence for confounds with other variables, we cannot rule out this possibility. Other investigators have reported increased vulnerability for African Americans compared with Whites treated with FGAs (Glazer et al, 1993, 1994; Morgenstern and Glazer, 1993), which was not accounted for by confounds with dosage or other factorsSchneider et al, 2005). Higher prevalence rates for women have been frequently, although not consistently, reported, with sex differences most often found for samples of older people (Yassa and Jeste, 1997). However, none of the previous prospective studies of FGAs, including our own, reported higher incidence rates for women, (Glazer et al, 1999; Woerner et al, 1998; Morgenstern and Glazer, 1993; Chakos et al, 1996) and one reported higher rates for men in an elderly sample (Yassa et al, 1992). Nevertheless, our study is relatively unique in that $70 \%$ of the participants were females, which is in contrast to an almost similar predominance of male schizophrenia trials and which possibly enhanced our power to assess TD risk in females. We have no explanation for the finding of decreased vulnerability on the part of patients treated with antidepressants prior to the study, and know of no similar findings in the literature.

Finally, we found that any exposure to an FGA (usually haloperidol, and by study design always for a maximum of 13 days) increased the risk of TD. We cannot rule out the possibility that this could be explained by other (confounding) variables such as medication dosage. If confirmed, this finding would indicate the need to modify the common clinical practice of prescribing PRN haloperidol to treat agitation in geriatric patients. A recent report indicates that there is still considerable use of FGAs among older people. Based on data for 2.5 million Medicare beneficiaries in nursing homes during 2001-2002, Briesacher et al (2005) reported that an estimated $27.6 \%$ of all Medicare beneficiaries were prescribed an antipsychotic drug at least once; about one quarter of them $(7.3 \%$ of all Medicare beneficiaries) received an FGA.
The findings of this study have to be interpreted within its limitations. These include the non-randomized, observational cohort study design, lack of a placebo or FGA control group, insufficient data to calculate cumulative antipsychotic dose, and modest sample size. Moreover, although $79 \%$ of the patients had psychosis, $59 \%$ had dementia and $31 \%$ had mood disorders. Therefore, the TD risk needs to be extrapolated to patients with schizophrenia. Nevertheless, the inclusion of almost a third of patients with mood disorders is relevant as this population is increasingly treated with antipsychotics. Despite these limitations, this is the largest prospective study of the two SGAs risperidone and olanzapine in antipsychotic-naïve, elderly patients who were followed for 2 years and rigorously assessed for the development of TD. The results from this study are especially informative for two reasons. First, data were collected in an exquisitely vulnerable elderly patient population with a mean age of 80 years in whom as little as 1 month of antipsychotic exposure suffices to result in TD. As a result, an informative study of a longer term side effect could be completed in a reasonable time span and the data are relevant to all patients, not just the elderly, in whom the risk is only accentuated. Second, studying antipsychotic-naïve subjects and careful attention (and analysis) of a cross-over to FGAs after the start of the study allowed us to attribute TD to the treatments being studied prospectively. These data are clinically meaningful, given the demographic shift in the US and other developed countries around the world that is associated with an increasing prevalence of disorders where antipsychotic medications are being used.

In conclusion, our data indicate that the enthusiasm expressed by the field for the relative safety of the SGAs, in terms of TD, is warranted. However, the risk is not zero, thus caution is still indicated, perhaps especially for women and African Americans. In addition, recent concerns about increased cardiovascular and cerebrovascular morbidity and mortality associated with SGAs (Ray et al, 2009; Schneider et al, 2005; Wang et al, 2005) underscores the need for a cautious weighing of the benefits against the potential risks of antipsychotic use in older people.

\section{ACKNOWLEDGEMENTS}

This study was supported in part by NIMH Grant $\mathrm{MH}$ 32369, an NIMH Clinical Research Center grant (MH 41960), and an NIMH Intervention Research Center grant (MH 60575) to The Zucker Hillside Hospital. We are grateful to Ann Fletcher, DiAnn Shortell-Jacobson, Donna O'Shea, MA, Erica Greenberg, MA, and Natasha Bennett, MA for participation in data collection, and Joseph Bonavisa, Ronald Brenner, MD, Edward Eschmann, Howard Guzik, MD, Lucy Macina, MD, Mary Toland, RN, and the Geriatric Psychiatry staff at The Zucker Hillside Hospital for assistance in patient recruitment. We thank Delbert Robinson, MD, for helpful comments on the work. Eli Lilly and Company and Janssen Pharmaceuticals provided medication for patients who were prescribed one of the study drugs by their treating doctor but could not afford to pay for it. 


\section{DISCLOSURE}

Dr Correll has been a consultant and/or advisor to, or has received honoraria from Actelion, AstraZeneca, BoehringerIngelheim, Bristol-Myers Squibb, Cephalon, Eli Lilly, IntraCellular Therapies, Ortho-McNeill/Janssen/J\&J, GSK, Hoffmann-La Roche, Medicure, Otsuka, Pfizer, ScheringPlough, Supernus, Takeda, and Vanda. Dr Alvir is currently an employee of Pfizer Dr Kane has been a consultant and/or advisor to, or has received honoraria from Merck, Lundbeck, Novartis, Sepracor, Targacept, Esai, HoffmanLaRoche, Astra-Zeneca, Janssen, Pfizer, Eli Lilly, BristolMyers Squibb, Dainippon Sumitomo, Johnson \& Johnson, Otsuka, Vanda, Proteus, Takeda, Targacept, IntraCellular Therapies, Rules Based Medicine, Boehringer-Ingelheim, and Sunovion. Drs Woerner, Greenwald, and Delman have nothing to disclose.

\section{REFERENCES}

American Psychiatric Association Committee on Nomenclature and Statistics (1987). Diagnostic and Statistical Manual of Mental Disorders, DSMIV. American Psychiatric Association: Washington, DC.

Briesacher BA, Limcango MR, Simone-Wastila L, Doshi JA, Levens SR, Shea DG et al (2005). The quality of drug prescribing in nursing homes. Arch Intern Med 165: 1280-1285.

Caroff S, Mann S, Campbell ES, Sullivan KA (2002). Movement disorders associated with atypical antipsychotic drugs. J Clin Psychiatry 63(Suppl 4): 12-19.

Casey DE (1999). Tardive dyskinesia and atypical antipsychotic drugs. Schizophrenia Res 35: S61-S66.

Chakos MH, Alvir JMaJ, Woerner MG, Koreen A, Geisler S, Mayerhoff D et al (1996). Incidence and correlates of tardive dyskinesia in first episode of schizophrenia. Arch Gen Psychiatry 53: 313-319.

Correll CU, Leucht S, Kane JM (2004). Lower risk for tardive dyskinesia associated with second-generation antipsychotics: a systematic review of 1-year studies. Am J Psychiatry 161: 414-425.

Correll CU, Kane JM (2007). One-year incidence rates of tardive dyskinesia in children and adolescents treated with secondgeneration antipsychotics: a systematic review. J Child Adolesc Psychopharmacol 17: 647-656.

Correll CU, Schenk EM (2008). Tardive dyskinesia and new antipsychotics. Curr Opin Psychiatry 21: 151-156.

Davidson M, Harvey PD, Vervarcke J, Gagiano CA, De Hooge JD, Bray G, Risperidone Working Group et al (2000). A long-term, multicenter, open-label study of risperidone in elderly patients with psychosis. Int J Geriatr Psychiatry 15: 506-514.

de Leon J (2007). The effect of atypical vs typical antipsychotics on tardive dyskinesia: a naturalistic study. Eur Arch Psychiatry Clin Neurosci 257: 169-172.

Dolder CR, Jeste DV (2003). Incidence of tardive dyskinesia with typical $v s$ atypical antipsychotics in very high risk patients. Biol Psychiatry 53: 1142-1145.

Glazer WM, Morgenstern H, Doucette JT (1993). Predicting the long-term risk of tardive dyskinesia in outpatients maintained on neuroleptic medications. J Clin Psychiatry 54: 133-139.

Glazer WM, Morgenstern H, Doucette J (1994). Race and tardive dyskinesia among outpatients at a CMHC. Hosp Commun Psychiatry 45: 38-42.

Glazer WM, Morgenstern H, Pultz JA, Yeung PP, Rak IW (1999). Incidence of persistent tardive dyskinesia may be lower with quetiapine treatment than previously reported with typical antipsychotics in patients with psychoses. In: Proceedings of the 38th Annual Meeting of the American College of Neuropsychopharmacology. ACNP: Nashville, TN. p 271.

Guy W (1976). ECDEU Assessment Manual for Psychopharmacology, revised. National Institute of Mental Health: Rockville, MD. Pub. No. (ADM) 76-338. National Institute of Mental Health.

Jeste DV, Caliguiri MP, Paulsen JS, Heaton RK, Lacro JP, Harris MJ et al (1995). Risk of tardive dyskinesia in older patients: a prospective longitudinal study of 266 outpatients. Arch Gen Psychiatry 52: 756-765.

Jeste DV, Lacro JP, Palmer B, Rockwell E, Harris MJ, Caligiuri MP (1999a). Incidence of tardive dyskinesia in early stages of low-dose treatment with typical neuroleptics in older patients. Am J Psychiatry 156: 309-311.

Jeste DV, Okamoto A, Napolitano J, Kane JM, Martinez RA (2000). Low incidence of persistent tardive dyskinesia in elderly patients with dementia treated with risperidone. Am J Psychiatry 157: $1150-1155$.

Jeste DV, Rockwell E, Harris MJ, Lohr JB, Lacro J (1999b). Conventional vs newer antipsychotics in elderly patients. Am J Geriatr Psychiatry 7: 70-76.

Kane JM, Woerner MG, Pollack S, Safferman AZ, Lieberman JA (1993). Does clozapine cause tardive dyskinesia? J Clin Psychiatry 54: 327-330.

Kinon BJ, Stauffer VL, Kaiser CJ, Hay DP, Kollack-Walker S (2005). Incidence of presumptive tardive dyskinesia in elderly patients treated with olanzapine or conventional antipsychotics. Poster Presented at the International Congress of Parkinson's Disease and Movement Disorders: New Orleans, Louisiana, USA. Poster no. 49.

Lee PE, Sykora K, Gill SS, Mamdani M, Marras C, Anderson G et al (2005). Antipsychotic medications and drug-induced movement disorders other than Parkinsonism: a populationbased cohort study in older adults. J Am Geriatr Soc 53: 1374-1379.

Marder SR, Essock SM, Miller AL, Buchanan RW, Davis JM, Kane JM et al (2002). The Mount Sinai Conference on the pharmacotherapy of schizophrenia. Schizophr Bull 28: 5-16.

Miller DD, Caroff SN, Davis SM, Rosenheck RA, Mc E, Saltz BL et al (2008). Extrapyramidal side-effects of antipsychotics in a randomised trial. Br J Psychiatry 193: 279-288.

Morgenstern H, Glazer WM (1993). Identifying risk factors for tardive dyskinesia among neuroleptic medication: results of the Yale tardive dyskinesia study. Arch Gen Psychiatry 50: 723-733.

Peduzzi P, Concato J, Kemper E, Holford TR, Feinstein AR (1996). A simulation study of the number of events per variable in logistic regression analysis. J Clin Epidemiol 49: 1373-1379.

Ray WA, Chung CP, Murray KT, Hall K, Stein CM (2009). Atypical antipsychotic drugs and the risk of sudden cardiac death. N Engl $J$ Med 360: 225-235. Erratum in $N$ Engl J Med. 2009 Oct 29;361(18):1814.

Saltz BL, Woerner MG, Kane JM, Lieberman JA, Alvir JM, Bergmann KJ et al (1991). Prospective study of tardive dyskinesia incidence in the elderly. JAMA 266: 2402-2406.

SAS Institute (1989). SAS/STAT User's Guide, version 6, Vol 2, 4th edn. SAS Institute: Cary, NC.

SAS Institute (1994). SAS/STAT Software: Changes and Enhancements, SAS Institute: Cary, NC.

Schneider LS, Dagerman KS, Insel P (2005). Risk of death with atypical antipsychotic drug treatment for dementia: metaanalysis of randomized placebo-controlled trials. JAMA 294: 1934-1943.

Schooler NR, Kane JM (1982). Research diagnoses for tardive dyskinesia (RD-TD). Arch Gen Psychiatry 39: 486-487.

Simpson GM, Angus JW (1970). A rating scale for extrapyramidal side effects. Acta Psychiatr Scand Suppl 212: 11-19. 
TD rates with risperidone or olanzapine in the elderly MG Woerner et al

Simpson GM, Lee JH, Zoubok B, Gardos G (1979). A rating scale for tardive dyskinesia. Psychopharmacology 64: 171-179.

Wang PS, Schneeweiss S, Avorn J, Fischer MA, Mogun H, Solomon DH et al (2005). Risk of death in elderly users of conventional $v s$ atypical antipsychotic medications. $N$ Engl J Med 353: 2335-2341.

Woerner MG, Alvir JMaJ, Saltz BL, Lieberman JA, Kane JM (1998). Prospective study of tardive dyskinesia in the elderly: rates and risk factors. Am J Psychiatry 155: 1521-1528.

Woods SW (2003). Chlorpromazine equivalent doses for the newer atypical antipsychotics. J Clin Psychiatry 64: 663-667.
Woods SW, Morgenstern H, Saksa JR, Walsh BC, Sullivan MC, Money $\mathrm{R}$ et al (2010). Incidence of tardive dyskinesia with atypical $v s$ conventional antipsychotic medications: a prospective cohort study. J Clin Psychiatry 71: 463-474.

Yassa R, Jeste DV (1997). Gender as a factor in the development of tardive dyskinesia. In: Yassa R, Nair NPV, Jeste DV (eds). Neuroleptic Induced Movement Disorder. Cambridge University Press: Cambridge, 40-56.

Yassa R, Nastase C, Dupont D, Thibeau M (1992). Tardive dyskinesia in elderly psychiatric patients: a 5-year study. Am J Psychiatry 149: 1206-1211. 